[Supporting Information]

\title{
Wearable, Ultrawide-Range, and Bending- Insensitive Pressure Sensor based on Carbon Nanotube Network-Coated Porous Elastomer Sponges for Human Interface and Healthcare Devices
}

Seunghwan Kim, ${ }^{a}$ Morteza Amjadi, ${ }^{b}$ Tae-Ik Lee, ${ }^{a}$ Yongrok Jeong, ${ }^{a}$ Donguk Kwon, ${ }^{a}$ Min Seong Kim, ${ }^{a}$ Kyuyoung Kim, Taek-Soo Kim, ${ }^{a}$ Yong Suk Oh*,,${ }^{a, c}$ and Inkyu Park*, ${ }^{a}$

${ }^{a}$ Department of Mechanical Engineering, Korea Advanced Institute of Science and Technology (KAIST), 291 Daehak-ro, Yuseong-gu, Daejeon 305-701, South Korea

${ }^{\mathrm{b}}$ Physical Intelligence Department Max-Planck Institute for Intelligent Systems

Heisenbergstrasse 3, Stuttgart 70569, Germany

${ }^{\mathrm{c}}$ Center for Bio-Integrated Electronics (CBIE), Northwestern University, Evanston, Illinois 60208, United States

\section{Corresponding Author}

*E-mail: inkyu@kaist.ac.kr

*E-mail: oyongsuk@kaist.ac.kr 
Movie S1. Internal cross-sectional geometries investigated by X-ray micro CT, showing a consistency of the micropores over the entire volume of the CCPPS.

Movie S2. Gradual geometrical change of the microporous configuration of the CCPPS during several compression/releasing cycles (0-80\%).

Movie S3. Demonstration of a 1D sensor array

Movie S4. Demonstration of a playing performance of the flexible piano pad showing the capability of sound volume control, playing at a fast tempo, a series of musical chord implementation, and playing a song.

Movie S5. Demonstration of a real-time 3D plotting of the foot pressure distribution with changes in posture. 

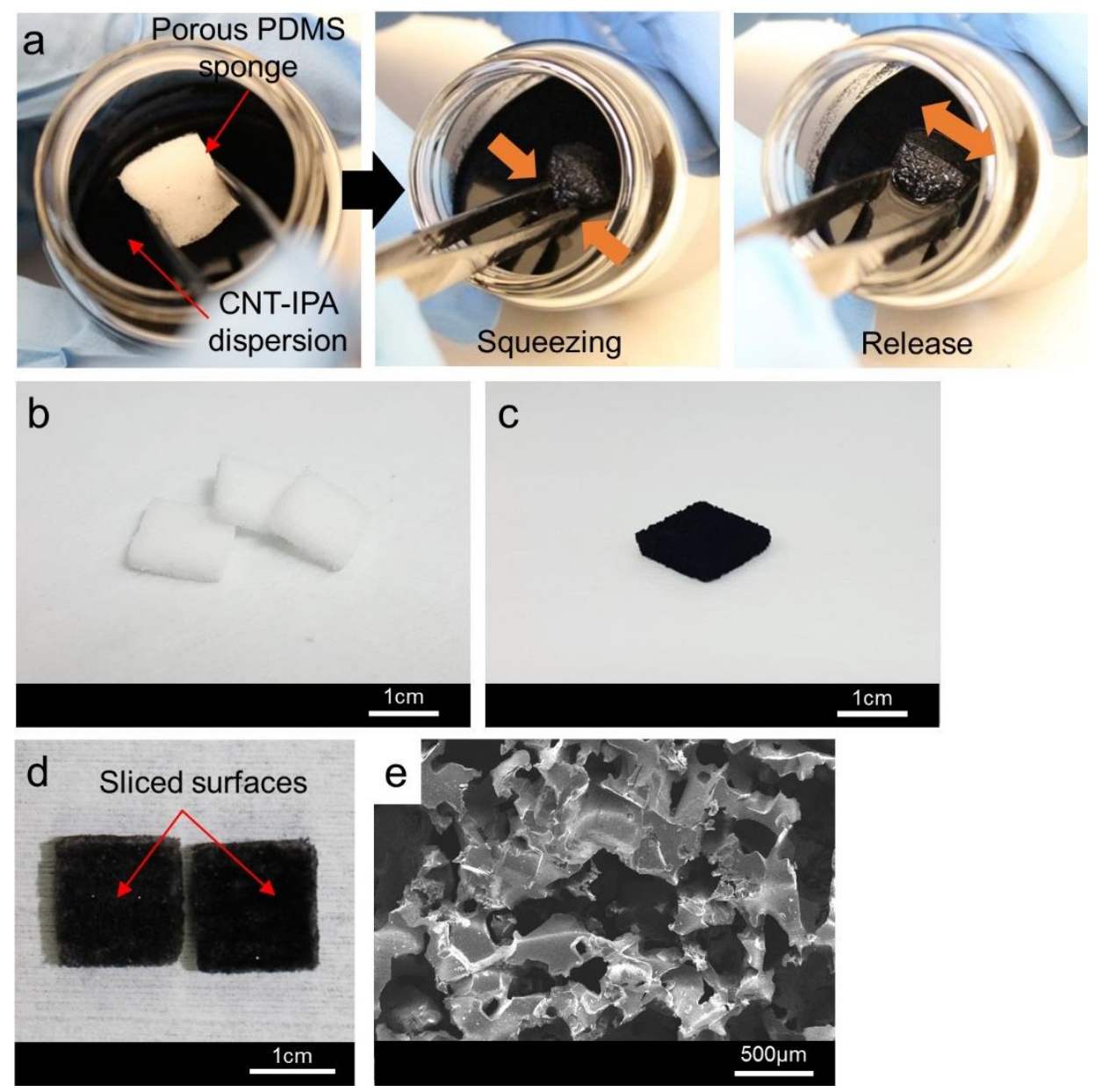

Figure S1. (a) Photographic image of a dip-coating method for the preparation of a CCPPS. The CCPPS was fabricated by dipping a PPS into CNT-IPA dispersion, followed by repeated squeezing. (b and c) Photographic images of the PPS with white color and the CCPPS with black color, respectively. (d) Photographic image of a slice of the CCPPS cut in half, showing that CNTs were evenly coated over the entire volume of the PPS. (e) SEM image of the surface morphology of the bare porous PDMS backbone. 


\section{Table S1. Porosity of the CCPPS}

\begin{tabular}{|c|c|c|c|}
\hline Sample \# & Porosity (\%) & Average (\%) & Standard deviation $(\%)$ \\
\hline 1 & 72.89 & \multirow{10}{*}{73.0} & \multirow{10}{*}{ \pm 0.59} \\
\hline 2 & 72.51 & & \\
\hline 3 & 73.24 & & \\
\hline 4 & 73.22 & & \\
\hline 5 & 71.98 & & \\
\hline 6 & 73.47 & & \\
\hline 7 & 72.14 & & \\
\hline 8 & 73.38 & & \\
\hline 9 & 73.37 & & \\
\hline 10 & 73.43 & & \\
\hline
\end{tabular}

It was assumed that the porosity of the CCPPS is same with that of the PPS, because the volume and mass of the CNTs can be negligible compared to those of the PDMS backbone. Ten samples were used to calculate the porosity as the following steps:

(1) Measurement of the mass and volume of the PPS: $m_{\mathrm{PPS}}, V_{\mathrm{PPS}}$

(2) Calculation of the volume occupied by the PDMS backbone except for the air in the PPS:

$V_{\mathrm{PPS}, \text { only PDMS }}=m_{\mathrm{PPS}} / \rho_{\mathrm{PDMS}}$

(3) Calculation of the porosity of the CCPPS (= PPS) using the ratio of $V_{\mathrm{PPS}}$ and $V_{\mathrm{PPS} \text {, only PDMS: }}$

Porosity $(\%)=\left(1-V_{\mathrm{PPS}, \text { only PDMS }} / V_{\mathrm{PPS}}\right) \times 100=73.0 \pm 0.59 \%$ 
a
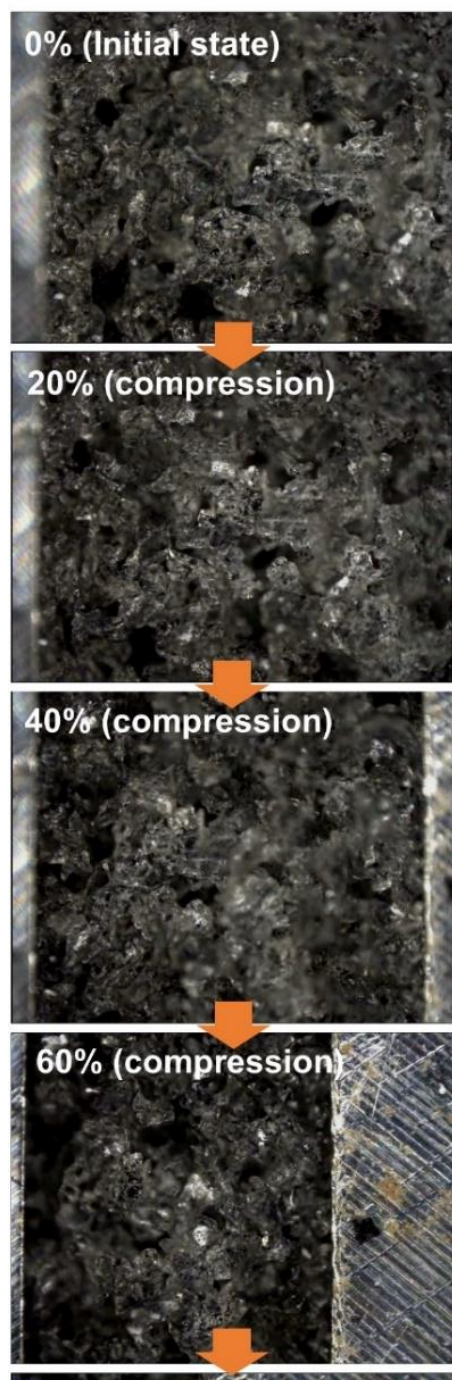

$80 \%$ (comphission)

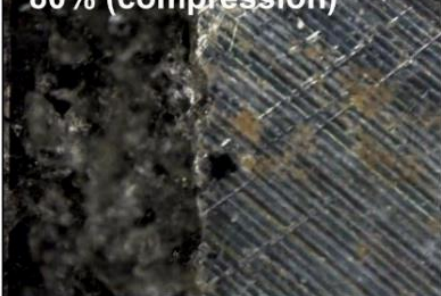

b
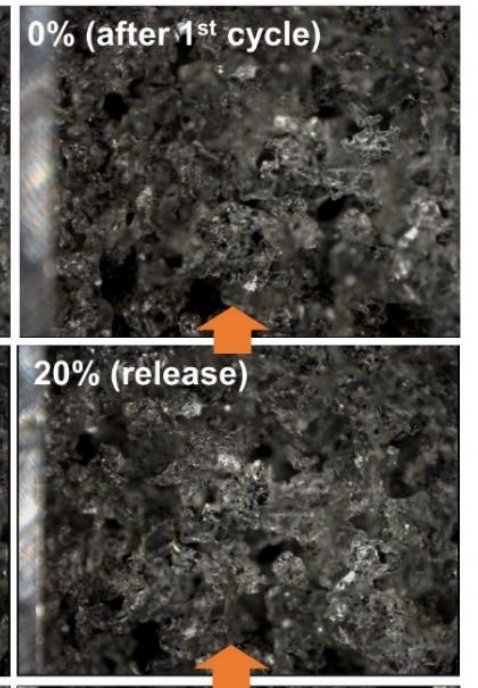
)

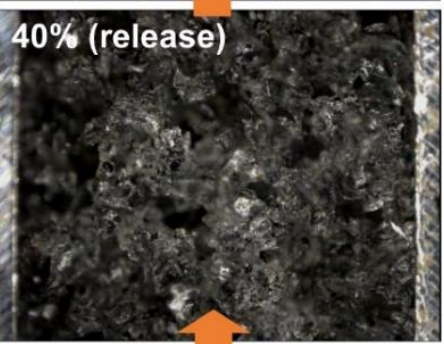

$60 \%$ (release)

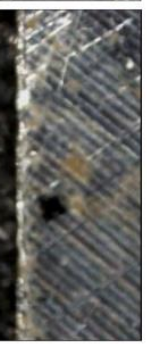

$\nabla$
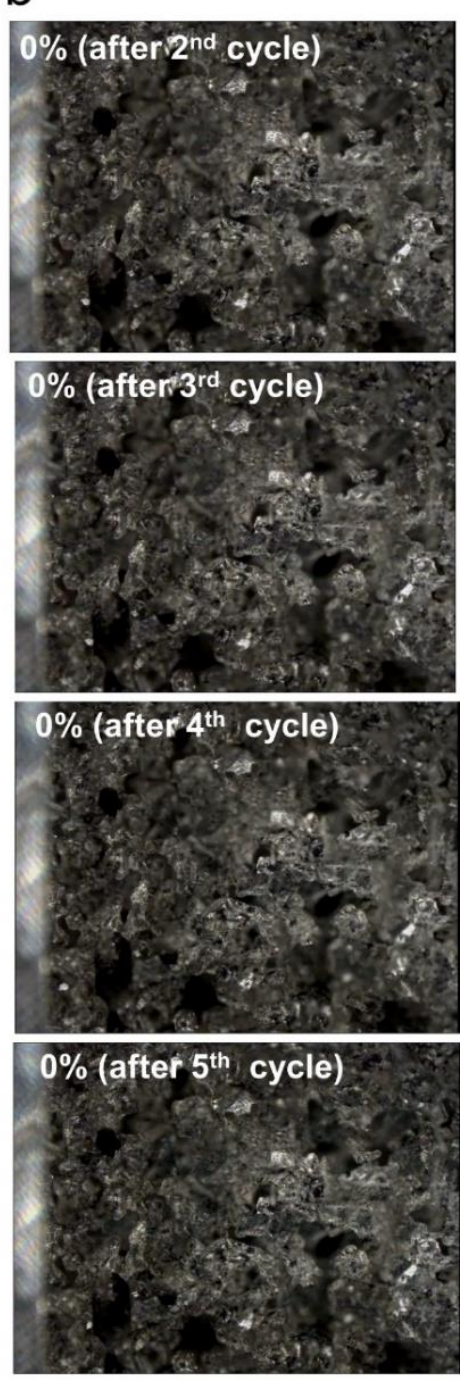

Figure S2. Optical images of a CCPPS (a) during a compression/release cycle (0-80-0\%) and (b) at $0 \%$ compression states after several compression/release cycles, showing reversible and repeatable closure/opening behavior of the micropores. 
a

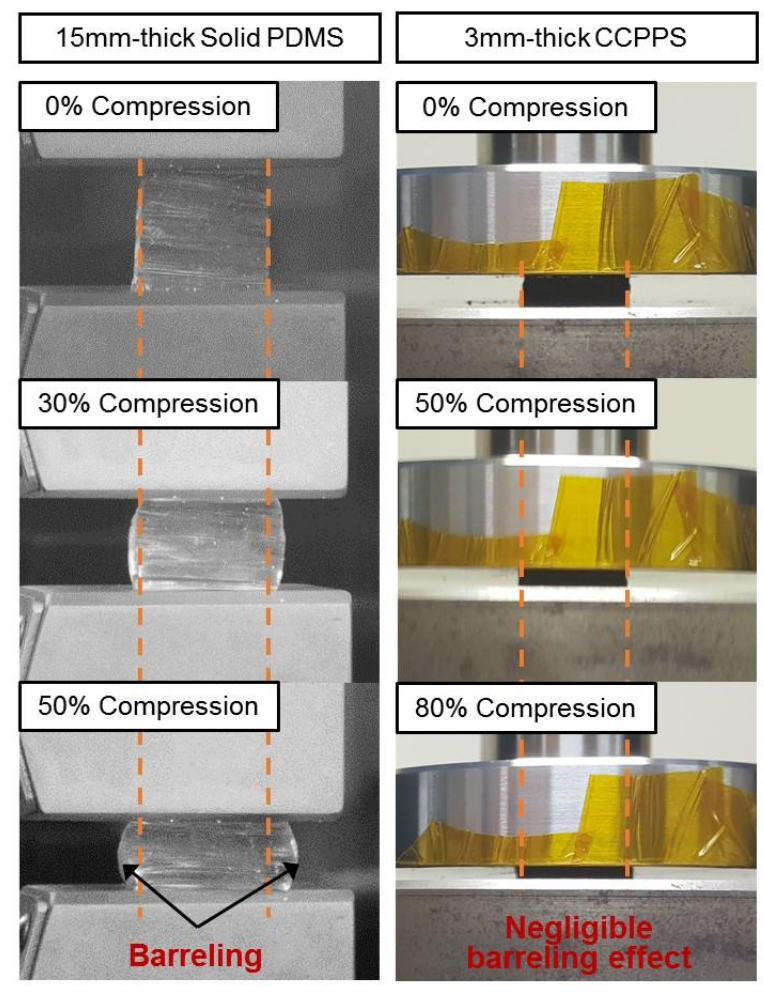

b
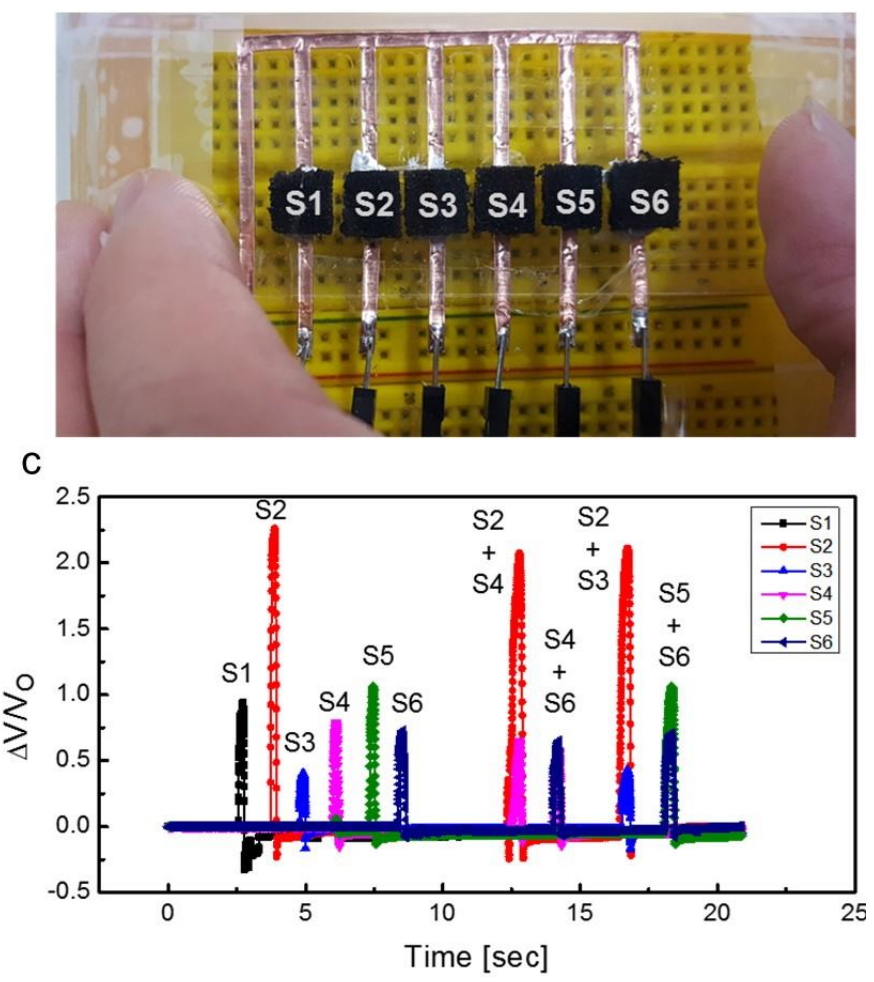

Figure S3. (a) Antibarreling property of a CCPPS. Unlike a solid PDMS, the CCPPS exhibits negligible lateral volume expansion a compression of $80 \%$. (b) Photographic image of the fabricated 1D sensor array. (c) Real-time measurement of the 6 sensor signals. Each sensor is able to accurately measure the local pressures without electrical and physical surrounding interference. 

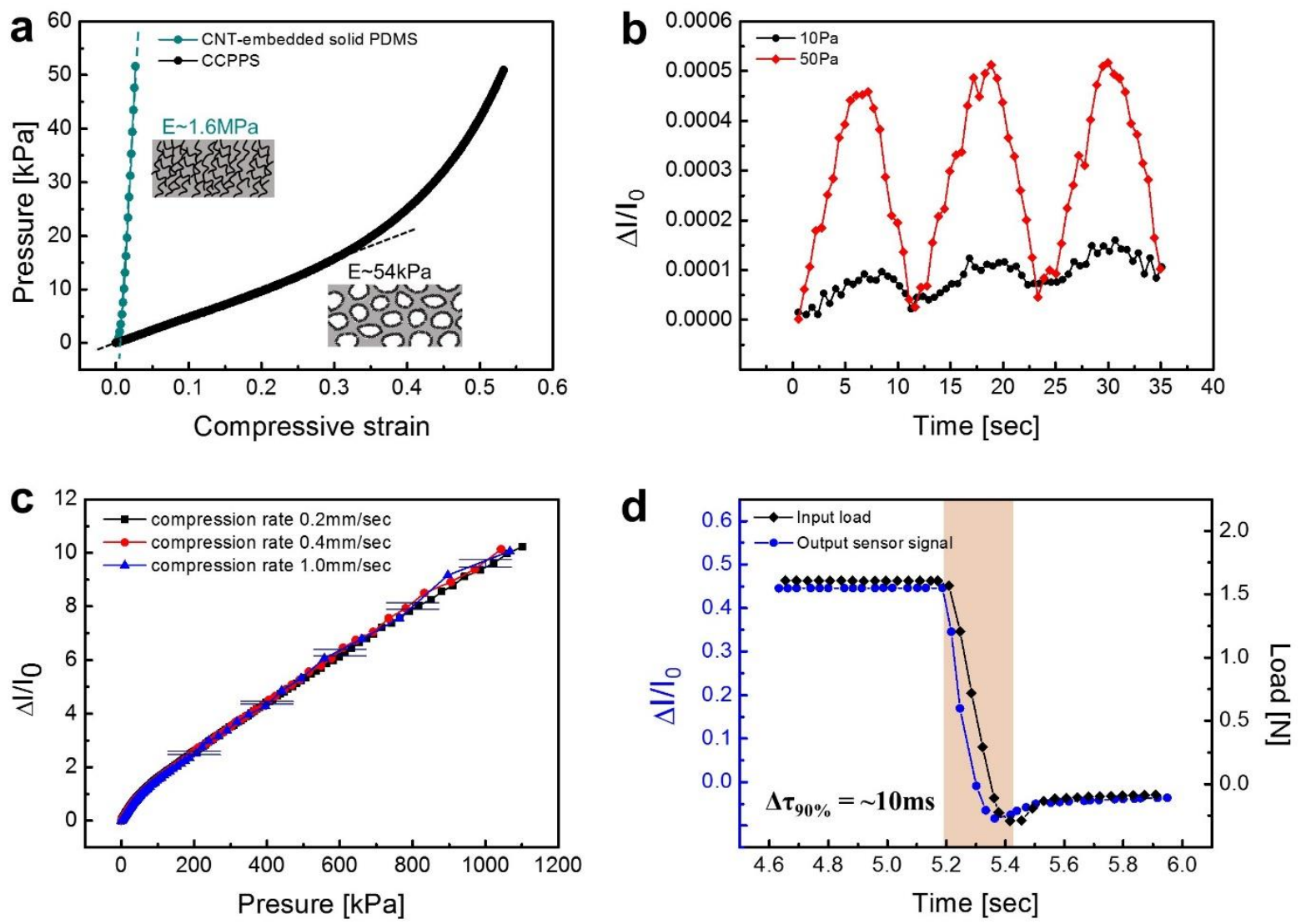

Figure S4. (a) Stress-strain curves of a CCPPS and a CNT-embedded solid PDMS in a low pressure range $(<50 \mathrm{kPa})$. (b) Detection of an ultralow pressure range $(10 \mathrm{~Pa}$ and $50 \mathrm{~Pa})$. (c) Pressure response curves at different compression speeds. (d) Transient response under a step unloading. 
a

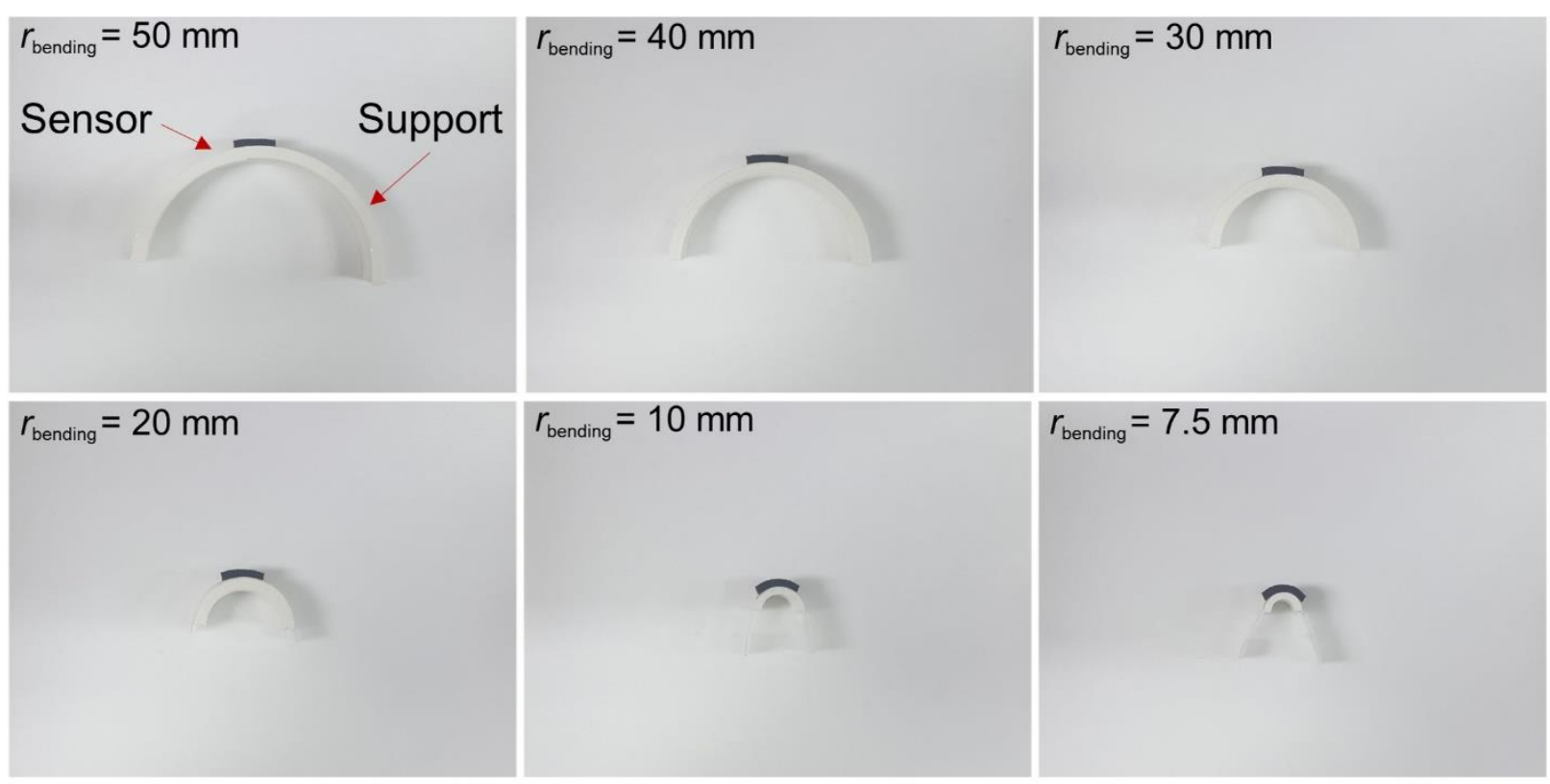

b
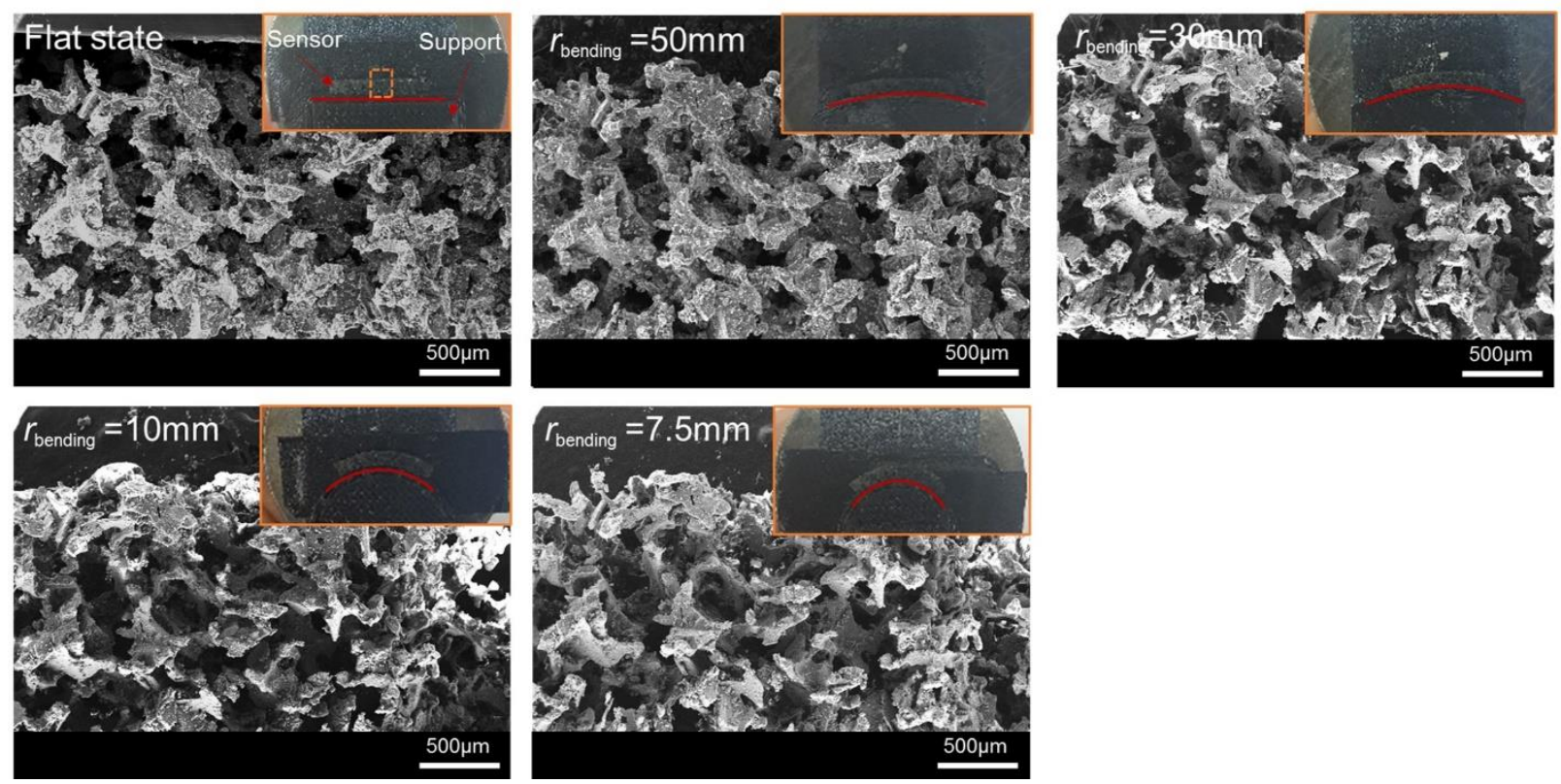

Figure S5. (a) Photographic images of CCPPS-based flexible pressure sensors attached to the curved surfaces of the 3D-printed supports with various bending radii. (b) Cross-sectional SEM images of the CCPPS under various bending radii. 

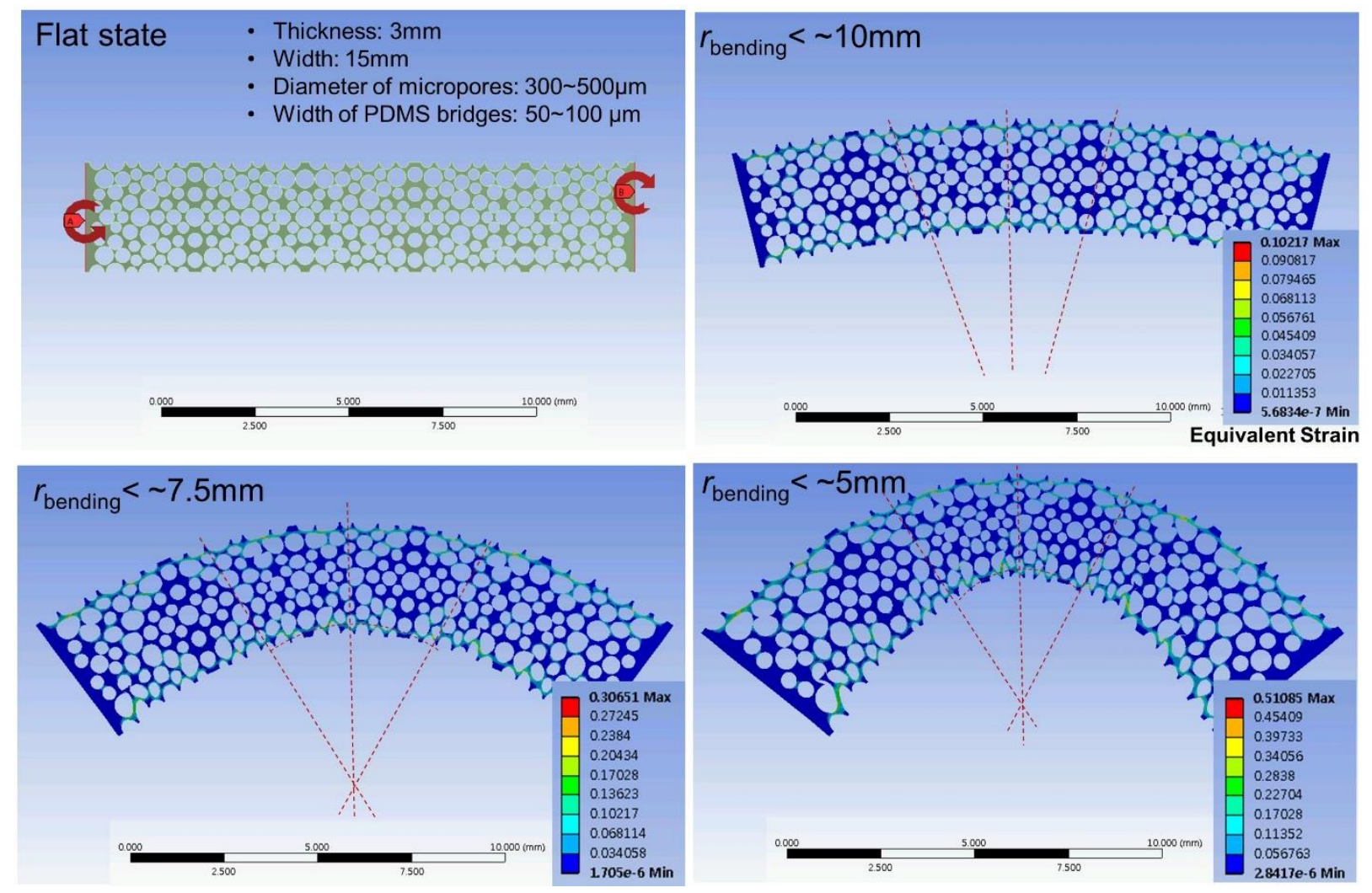

Figure S6. Simulation analysis of bending of a CCPPS. Bending insensitivity of the CCPPS was investigated using a commercial finite element analysis (FEA) software, ANSYS. Locations and dimensions of micropores were randomly distributed. The structure was modeled as a linearly elastic material. Thickness/width of the entire structure, size of the micropores, and thickness of PDMS bridges were modeled as the real scales of the CCPPS. Even under bending of the entire sensor structure, noticeable distortion or contact between the micropores causing the current change do not occur. 
a

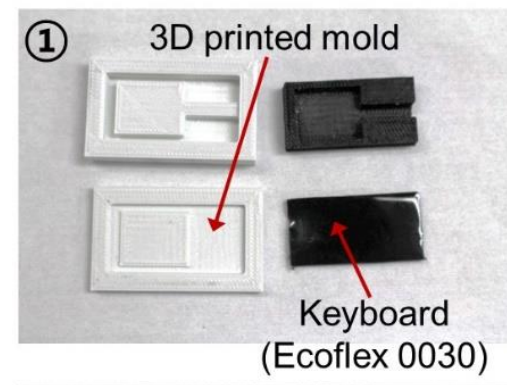

(2)
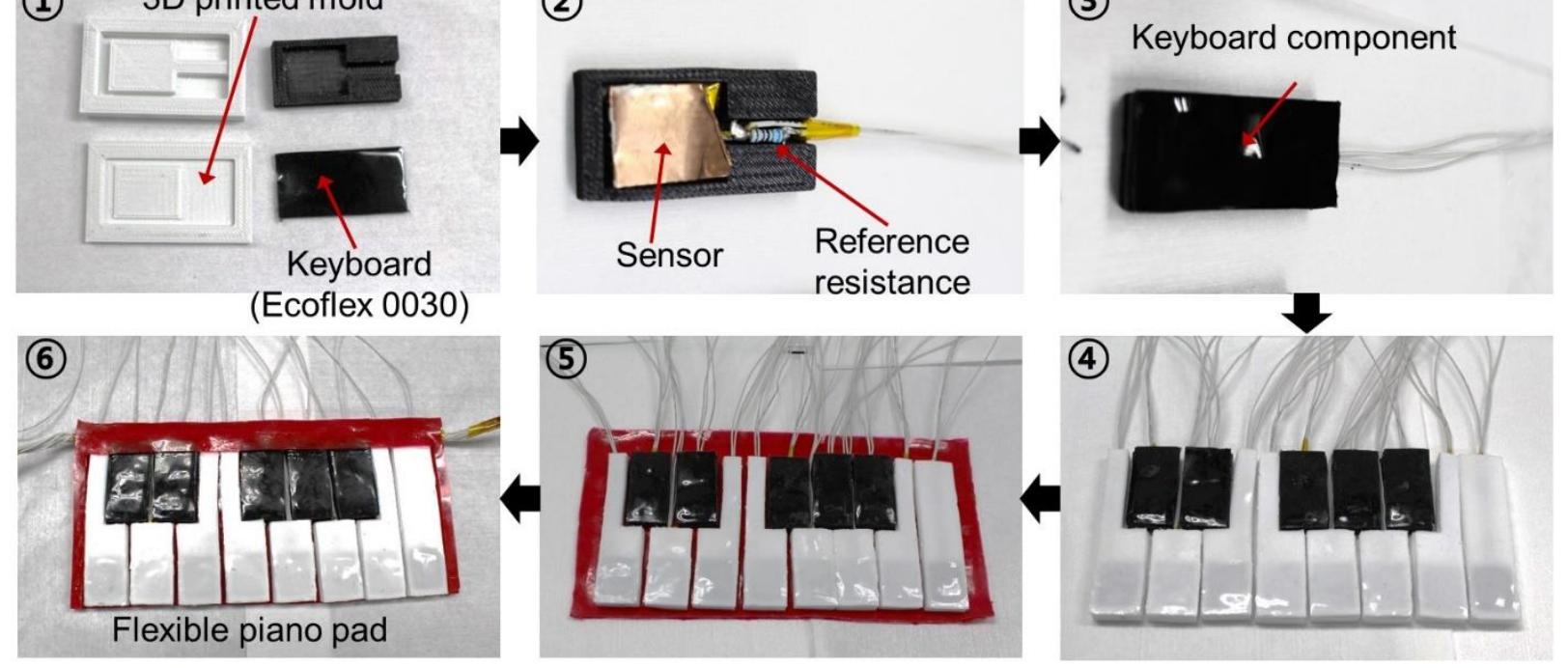

b

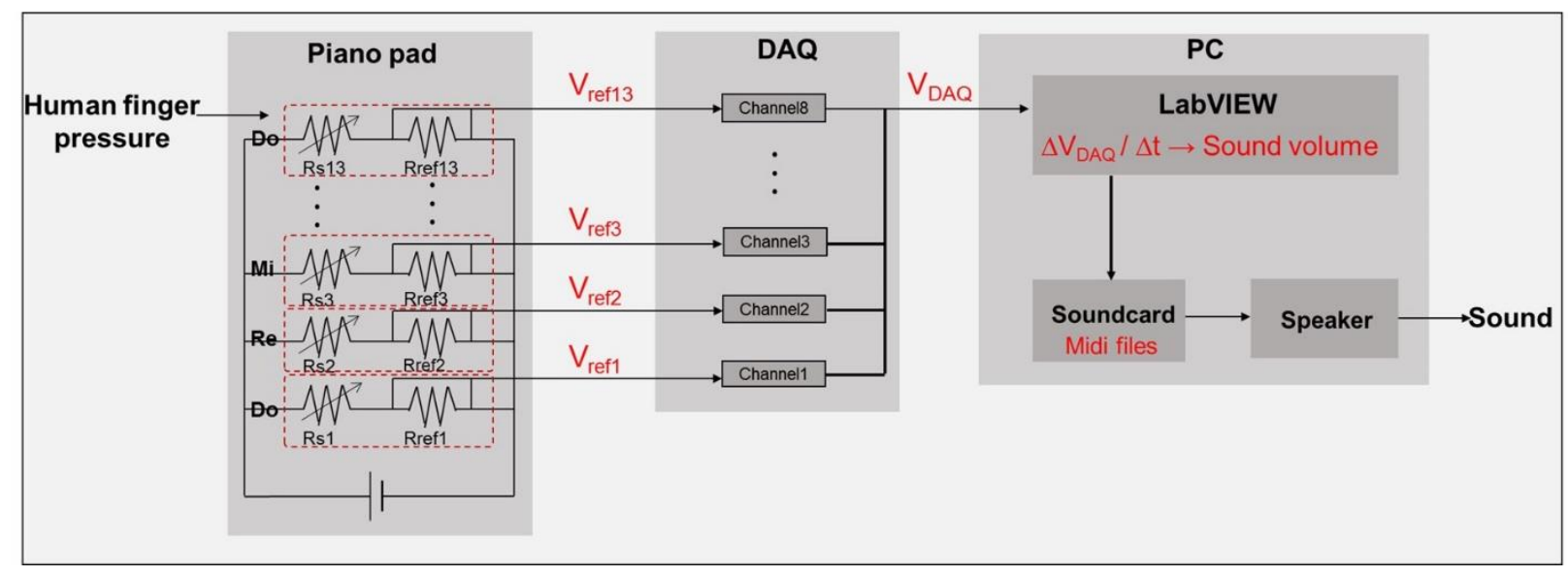

Figure S7. (a) Fabrication procedures of the flexible piano pad. (1) Fabrication of molds with a 3D

printer and keyboard housings made of soft elastomer (Ecoflex). (2) Formation of electrical circuits in the keyboards (3) Fabrication of the keyboard component. (4), (5) Fabrication of the keyboard arrays. (6) Completed flexible piano pad. (b) Overall flow of the conversion of the output signals to the sound volumes. 
a

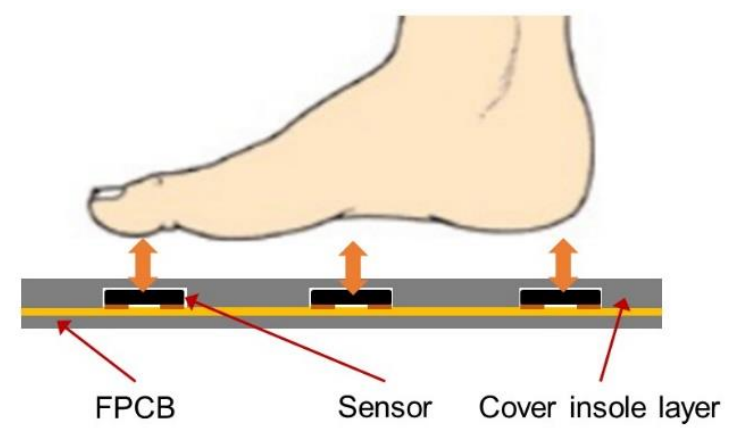

b

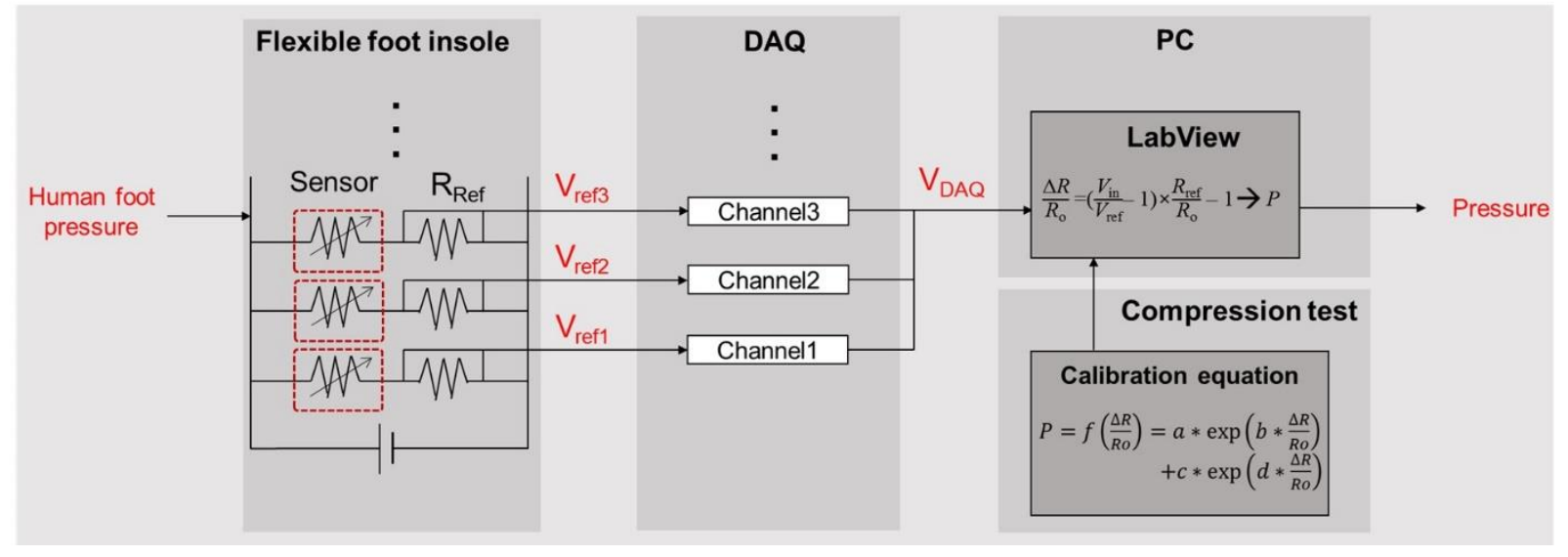

Figure S8. (a) Schematic illustration of cross-sectional structure of the flexible foot insole. The sensors were packaged in sockets of a cover layer. (b) Overall flow of detection of the foot pressure distribution. 